\title{
7. Conclusion
}

This review of experimental and theoretical work on the interaction of SPs with corrugated surfaces, which contains numerous interesting phenomena, has the intention of discussing the consistency of the material and discrepancies which demand reconsideration of the data. It is the hope that this review may induce experiments and calculations to clear up unanswered questions to settle the application of light scattering methods and to seek new viewpoints.

There is a rather good insight into the properties of the SPs concerning field enhancement, light emission from perturbated surfaces in the linear approximation. But since the limits of the validity of the first-order calculations are rather low, about $20 \AA$ for $\delta$ of the statistical roughness and about $70 \AA$ for the amplitudes $h$ of a sinusoidal profile, many experiments or calculations use roughness beyond these limits. It is therefore important to have a better insight as to the dependences of the enhancement, the light emission, and the dispersion relation on $\delta$ or $h$, above the linear approximation. The first steps are complete, but it needs much more reliable experimental data, to get a thorough understanding of the subject. 